\title{
Revealing the Nature of Photoluminescence Emission in Metal-Halide Double Perovskites
}

\author{
S. J. Zelewski, ${ }^{\dagger \ddagger}$ J. M. Urban, ${ }^{\dagger}$ A. Surrente, ${ }^{\dagger}$ D. K. Maude, ${ }^{\dagger}$ A. Kuc, ${ }^{\top}$ L. \\ Schade, ${ }^{\S}$ R. D. Johnson,,$^{\S}$ M. Dollmann, ${ }^{\S}$ P. K. Nayak, ${ }^{\S}$ H. J. Snaith,, P. G. \\ Radaelli, ${ }^{\S}$ R. Kudrawiec, ${ }^{\ddagger}$ R. J. Nicholas, ${ }^{\S}$ P. Plochocka, ${ }^{*, \dagger}$ and M. \\ Baranowski*,,$\ddagger$ \\ †Laboratoire National des Champs Magnétiques Intenses, UPR 3228, \\ CNRS-UGA-UPS-INSA, Grenoble and Toulouse, France \\ $\ddagger$ Department of Experimental Physics, Faculty of Fundamental Problems of Technology, \\ Wroclaw University of Science and Technology, Wroclaw, Poland \\ ФHelmholtz-Zentrum Dresden-Rossendorf, Abteilung Ressourcenökologie, Forschungsstelle \\ Leipzig, Permoserstr. 15, 04318, Leipzig, Germany \\ $\S$ University of Oxford, Clarendon Laboratory, Parks Road, Oxford, OX1 3PU, United
}

Kingdom

E-mail: paulina.plochocka@lncmi.cnrs.fr; michal.baranowski@lncmi.cnrs.fr

February 22, 2019

\section{Abstract}

Double perovskite crystals such as $\mathrm{Cs}_{2} \mathrm{AgBiBr}_{6}$ are expected to overcome the limitation of classic hybrid organic-inorganic perovskite crystals related to the presence of lead and the lack of structural stability. Perovskites are ionic crystals in which the carriers are expected to strongly couple to lattice vibrations. In this work we demonstrate that the photoluminescence (PL) emission in $\mathrm{Cs}_{2} \mathrm{AgBiBr}_{6}$ is strongly influenced by the strong electron-phonon coupling. Combining photoluminescence excitation (PLE) and Raman spectroscopy we show that the PL emission is related to a color center rather than a band-to-band transition. The broadening and the Stokes shift of the PL emission from $\mathrm{Cs}_{2} \mathrm{AgBiBr}_{6}$ is well explained using a Franck-Condon model with a Huang-Rhys factor of $S=11.7$ indicating a strong electronphonon interaction in this material.

Lead-halide perovskite crystals have emerged as promising materials for photovoltaic applications. After less than a decade of development perovskite solar cells already exceed $20 \%$ power conversion efficiency ${ }^{11}$ establishing solution-processable technology as a viable alternative or extension to silicon solar cells. ${ }^{213}$ This tremendous progress in perovskite-based photovoltaics is related to the unique combination of large optical absorption coefficient, long carrier lifetime and diffusion length, and low effective masses of carriers in these materials. ${ }^{4}$ 요 With their simple and cheap fabrication technology the lead-halide perovskites seems to be ideal candidates for photovoltaic and other light emitting applications. 10

Despite their superior properties, the leadhalide perovskites have drawbacks which hinder the deployment of this technology on a large scale. Lead-halide perovskites tend to degrade when exposed to moisture, heat, or prolonged illumination in air. ${ }^{12}$ Even in the most stable compounds the practical lifetime of solar cells is limited to weeks rather than years. ${ }^{13} \mathrm{In}$ addition, the presence of $\mathrm{Pb}$ in solar cells is envi- 
ronmentally undesirable, with the formation of $\mathrm{PbI}_{2}$ during the degradation of perovskites. $\frac{1415}{115}$

It is expected that the intrinsic limitations of lead-halide perovskites can be overcome by the heterovalent substitution of $\mathrm{Pb}^{2+}$ by pairs of cations with formal oxidation states +1 and +3 forming so-called "double perovskite" with general formula $\mathrm{A}_{2}^{I} \mathrm{~B}^{I} \mathrm{~B}^{I I I} \mathrm{X}_{6} \cdot \frac{[15] 16]}{16}$ Here $\mathrm{A}$ is a cation, for example $\mathrm{Cs}^{+}$, filling space between halide $(\mathrm{X}=\mathrm{Cl}, \mathrm{Br}, \mathrm{I})$ octahedral cages. The +1 and +3 cations alternate along the $x, y$ and $z$ axes in neighbouring halide cages. There are numerous combinations of $1+$ and $3+$ ions with a suitable electron configuration such as $\mathrm{Cu}^{+}, \mathrm{Ag}^{+}, \mathrm{Bi}^{3+}, \mathrm{Sb}^{3+}$, and $\mathrm{In}^{3+}$. Band structure calculations show that many of these compounds have a band gap in the range promising for solar cell applications. 17 Some of the possible compounds have already been synthesized corroborating theoretical predictions. ${ }^{18}$ For example silver-bismuth double perovskites are highly stable ${ }^{17 \mid 20}$ indirect band gap semiconductors with the absorption edge in the range of 1.8-2.2 eV $\left(\mathrm{Cs}_{2} \mathrm{AgBiBr}_{6}\right)^{20121 / 23}$ and $2.2-2.8 \mathrm{eV}$ $\left(\mathrm{Cs}_{2} \mathrm{AgBiCl}_{6}\right) .1720121$ Moreover, recent studies have shown that these materials are very well situated for the detection of high energy radiation $^{24 \mid 25}$ and white light emitters. 26

Metal halide perovskites are ionic crystals built from closed-shell rare-gas-like ions so that a strong interaction of the carriers with the lattice vibrations is expected. $\sqrt[27]{29}$ The importance of electron-phonon coupling has already been demonstrated for a variety of perovskite semiconductors. $\stackrel{8] 30+35}{[35}$ However, for double perovskites most studies have focused on structural, band gap and stability properties. The electron-phonon coupling has only received attention ${ }^{26 / 31 / 36}$ very recently even though this interaction is crucial for understanding the electrical and optical properties of perovskite crystals.

In this work we show that the photoluminescence (PL) emission in $\mathrm{Cs}_{2} \mathrm{AgBiBr}_{6}$ is dominated by a strong electron-phonon interaction. Using a combination of PL, Raman and photoluminescence excitation (PLE) we reveal that the $\mathrm{PL}$ emission is related to a color center rather than a band-to-band tran- sition. The resonant excitation of this center results in a strong enhancement of PL emission demonstrating the importance of the nonradiative recombination path related to the indirect bandgap. Crucially, we show that the significant PL broadening together with the large Stokes shift between emission (PL) and absorption (PLE) can be well explained using a configurational coordinate diagram (FranckCondon model) indicating a strong electronphonon coupling characterized by a relatively large Huang-Rhys factor $\simeq 12$.

Figure 11 (a) shows the PL (red), PLE (blue) and reflectance (black line) spectra of $\mathrm{Cs}_{2} \mathrm{AgBiBr}_{6}$ single crystal taken at $T=5 \mathrm{~K}$. The PL emission, centered around $2 \mathrm{eV}$ is strongly broadened, with a full width half maximum $(\mathrm{FWHM})$ of $\simeq 190 \mathrm{meV}$. The PLE spectrum shows that the intensity of the PL emission strongly depends on the energy of the exciting photons. For excitation below $2.4 \mathrm{eV}$ the PL emission is almost completely quenched. For excitation above $2.4 \mathrm{eV}$ the PL signal increases rapidly reaching a maximum at around $2.5 \mathrm{eV}$ before decreasing slowly at higher energies. A sharp decrease of PLE signal is observed around $2.8 \mathrm{eV}$ corresponding to the direct band gap exciton which is clearly visible as a resonance in the reflectance spectrum (see black lines in Fig. 1 (a)). ${ }^{37}$ This behaviour is unusual for band-to-band transitions where the PLE signal normally shows a monotonic increase $^{38}$ simply following the increasing density of states of the bands before eventually saturating for strong enough absorption. The complex dependence of PLE signal is in contrast to published absorption measurements 16 [23/39|40 and our photoacoustic spectrum (equivalent to absorption) presented in Fig.1 (b) which all exhibit monotonic behaviour. The onset of PLE is at hundreds of meV higher than the reported bandgap for $\mathrm{Cs}_{2} \mathrm{AgBiBr}_{6} \cdot \frac{|20| 21|23| 39}{}$

The non-monotonic behaviour of the PLE signal indicates that the observed PL emission is not related to a band-to-band transition but its origin can be attributed to a color center which can be effectively excited by $2.5 \mathrm{eV}$ photons. Excitation below this resonant energy results in a quenching of the PL signal since the 

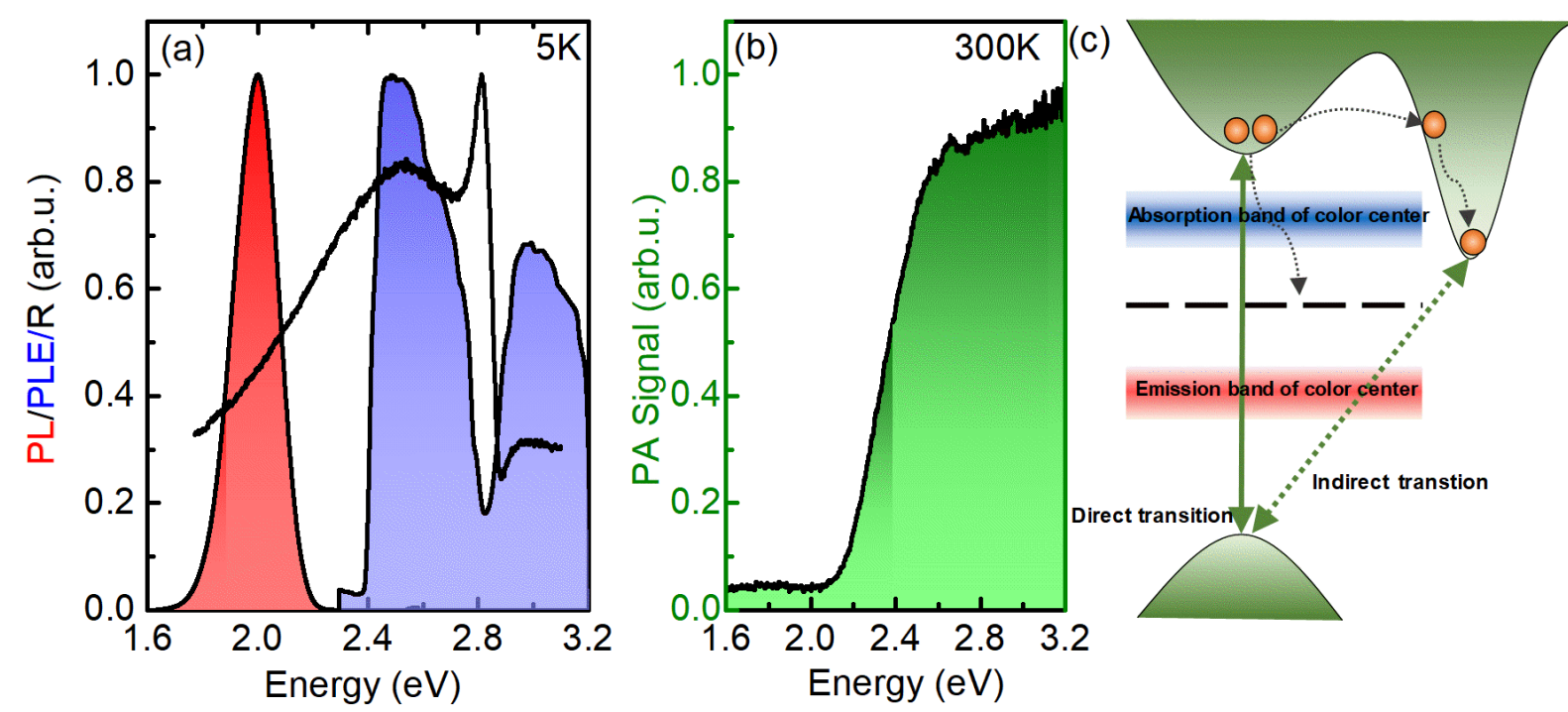

Figure 1: (a) Low temperature $(T=5 \mathrm{~K})$ photoluminescence spectrum (PL in red) and integrated $\mathrm{PL}$ intensity as a function of the energy of the exciting photons (PLE spectrum in blue) together with the reflectance spectrum ( $\mathrm{R}$ in black). (b) photoacoustic signal (PAS) as a function of the energy of excitation (equivalent to absorption) measured at $300 \mathrm{~K}$. (c) Schematic showing the band structure of $\left(\mathrm{Cs}_{2} \mathrm{AgBiBr}_{6}\right)$ with possible transitions and carrier relaxation pathways. The black dashed line indicates the position of color centers. The blue and red bars represent absorption and emission bands of the color center which are broadened and shifted in energy due to the strong electron-phonon coupling (see text).

emitting states are not populated/excited. This can be understood as a competition between recombination pathways; the carriers can either be trapped by the color centers or they can relax to the minimum of the indirect band gap as shown schematically in Fig. 11(c). The density of color centers and their photon absorption cross section cannot be very high since they are not seen in the absorption spectrum. This suggests that the carriers mostly relax to the minimum of the conduction band corresponding to indirect band gap where they recombine non-radiatively. This scenario of carrier relaxation and recombination is in agreement with the recent studies of PL and transient absorption dynamics which show that majority of the carriers relax to the indirect band gap states before recombining non-radiatively. 40141

In ionic crystals color centers are usually strongly coupled to the crystal lattice vibrations. ${ }^{27}$ The occupation of such a center causes a local lattice deformation leading to an energy offset between the ground (unoccupied) and excited (occupied) states of the color center. Ra- diative recombination can then occur accompanied by the emission of multiple phonons. ${ }^{27 / 42}$ This leads to a broadening of the absorption and emission bands, which are energetically separated as shown schematically in Fig. 1 (c)). The observed $\simeq 500 \mathrm{meV}$ Stokes shift between emission (PL) and absorption (PLE) together with the significant broadening of the PL emission are the smoking gun signature of a strong electron-phonon coupling in $\mathrm{Cs}_{2} \mathrm{AgBiBr}_{6}$.

The strength of the electron-phonon interaction is characterized by the Huang-Rhys factor $S$ which is the average number of phonons emitted by the color center after photon absorption or emission. The phonon energy can be extracted from the Raman spectrum presented in Fig. 2, The low temperate Raman spectrum is dominated by the peak at $180 \mathrm{~cm}^{-1}$ and two less intense peaks can be seen at $139 \mathrm{~cm}^{-1}$ and $74 \mathrm{~cm}^{-1}$. We identify the origin of each peak comparing it with the predictions of DFT calculations (see supplementary information for DFT details) marked by the vertical dashed lines. The peak around $180 \mathrm{~cm}^{-1}$ is the $\mathrm{A}_{1 \mathrm{~g}}$ 


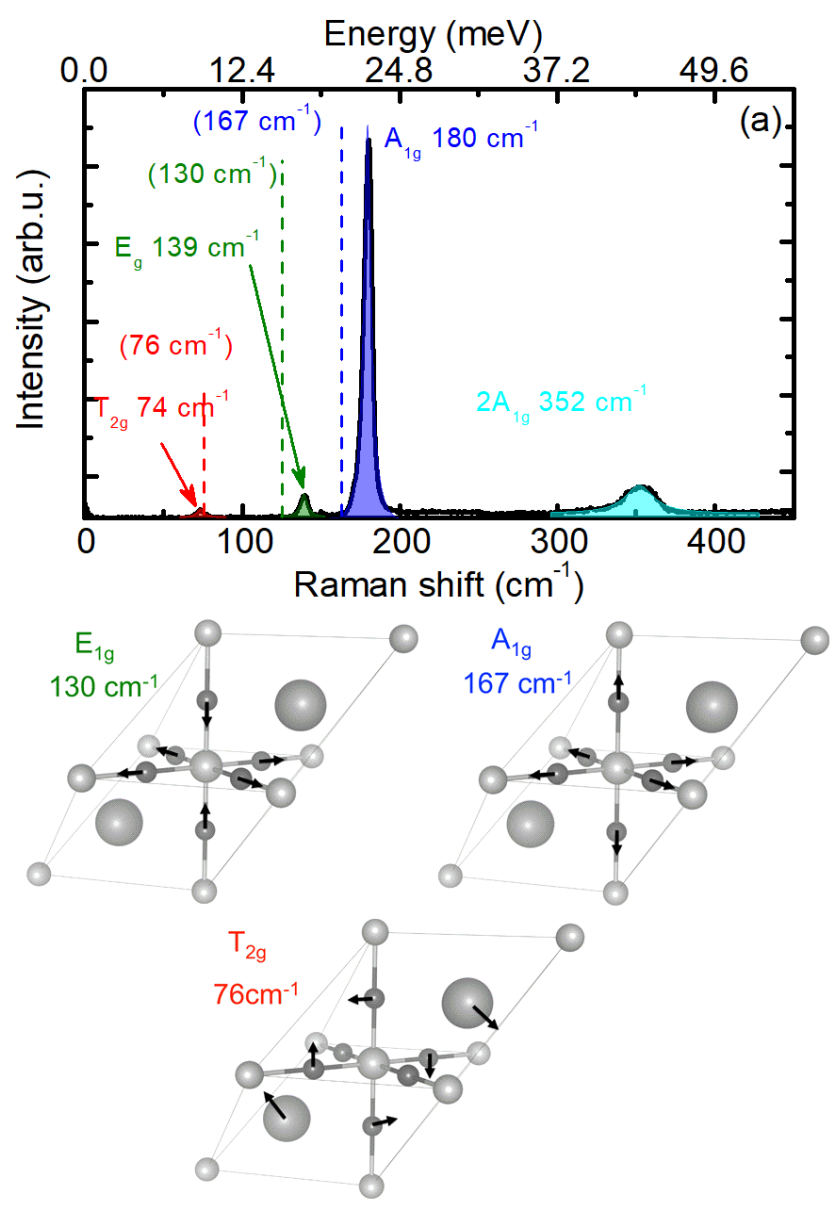

Figure 2: Low temperature $(5 \mathrm{~K})$ Raman spectrum of $\mathrm{Cs}_{2} \mathrm{AgBiBr}_{6}$. The experimental peaks are represented by solid lines while the Lorentzian fits are represented by shaded area. The dashed lines indicate the energy of vibration calculated using DFT.

LO phonon mode related to symmetric stretching vibration of $\mathrm{Br}$ atoms around $\mathrm{Bi}$ atoms in the octahedron and the two lower peaks are vibration with symmetries of $\mathrm{E}_{g}$ (at around 139 $\mathrm{cm}^{-1}$ ) and $\mathrm{T}_{2 g}$ (at around $74 \mathrm{~cm}^{-1}$ ). The $\mathrm{E}_{g}$ mode is related to the asymmetric stretching vibrations of $\mathrm{Br}$ around $\mathrm{Bi}$ atoms. The $\mathrm{T}_{2 g}$ mode is a correlated motion of Cs atoms with the scissoring of $\mathrm{Br}$ atoms (cf. Fig. 2). The calculated energies of the modes are slightly lower than measured experimentally, which might be related to disorder in $\mathrm{Ag}$ and Bi cation arrangement or a non fully relaxed crystal structure (the ionic radius of $\mathrm{Ag}$ is larger than that of $\mathrm{Bi}$, therefore bonding distortions arise due to the large mismatch of the two sublattices $\left.{ }^{\sqrt{31}}\right)$. The dependence of simulated Raman peaks on the lattice vectors is shown in Supporting Information (Fig. S2). The weak peak visible at around $350 \mathrm{~cm}^{-1}$ is usually attributed to the second order of the $\mathrm{A}_{1 g}$ mode, ${ }^{, 31}$ not observed in the DFT simulations.

According to a conventional Franck-Condon model the absorption and emission spectrum are composed of a series of transitions separated by the phonon energy $E_{p h}$. The intensity of each transition is proportional to the overlap of the harmonic oscillator states in the ground and excited states of the color center (see Fig 3 ). At low temperature only the ground vibrational state is occupied and the intensity of each transition is proportional to:

$$
I(n) \sim \frac{\mathrm{e}^{-S} S^{n}}{n !} .
$$

This predicts that the emission is a mirror image of the absorption spectrum. As the HuangRhys $S$ factor the absorption and emission spectrum change from being dominated by the 0 phonon line to multiple phonon excitation and emission. This is shown in Fig. 3 (b)-(d) where we calculate the emission and absorption for different values of $S$ and line widths of the individual phonon transitions (FWHM of $1 \mathrm{meV}$ (blue-red) and $10 \mathrm{meV}$ (lighter colored shaded area)). Typically, for strong electronphonon coupling the broadening of the phononassisted transitions results in smooth broad emission and absorption bands without any phonon structure. ${ }^{27}$ The size of the Stokes shift is also controlled by the value of $S$, and for smooth bands (individual transitions not resolved) its value is somewhere between $(2 S-$ 1) $E_{p h}$ and $2 S E_{p h} .{ }^{42}$ In perovskite crystals the carrier scattering is dominated by longitudinal optical (LO) phonons ${ }^{31}$ which correspond to an energy of $\sim 22.4 \mathrm{meV}\left(180 \mathrm{~cm}^{-1}\right.$ in the Raman spectrum) for the $\mathrm{Cs}_{2} \mathrm{AgBiBr}_{6}$. Assuming $E_{p h}=22.4 \mathrm{meV}$ the PL and dominant PLE onset are very well reproduced using a HuangRhys factor of $S=11.7$ (see Fig.3) and a 0phonon line energy of $2.25 \mathrm{eV}$. Crucially, the Huang-Rhys factor $S$ is the only fitting parameter and reproduces exactly the Stokes shift and 

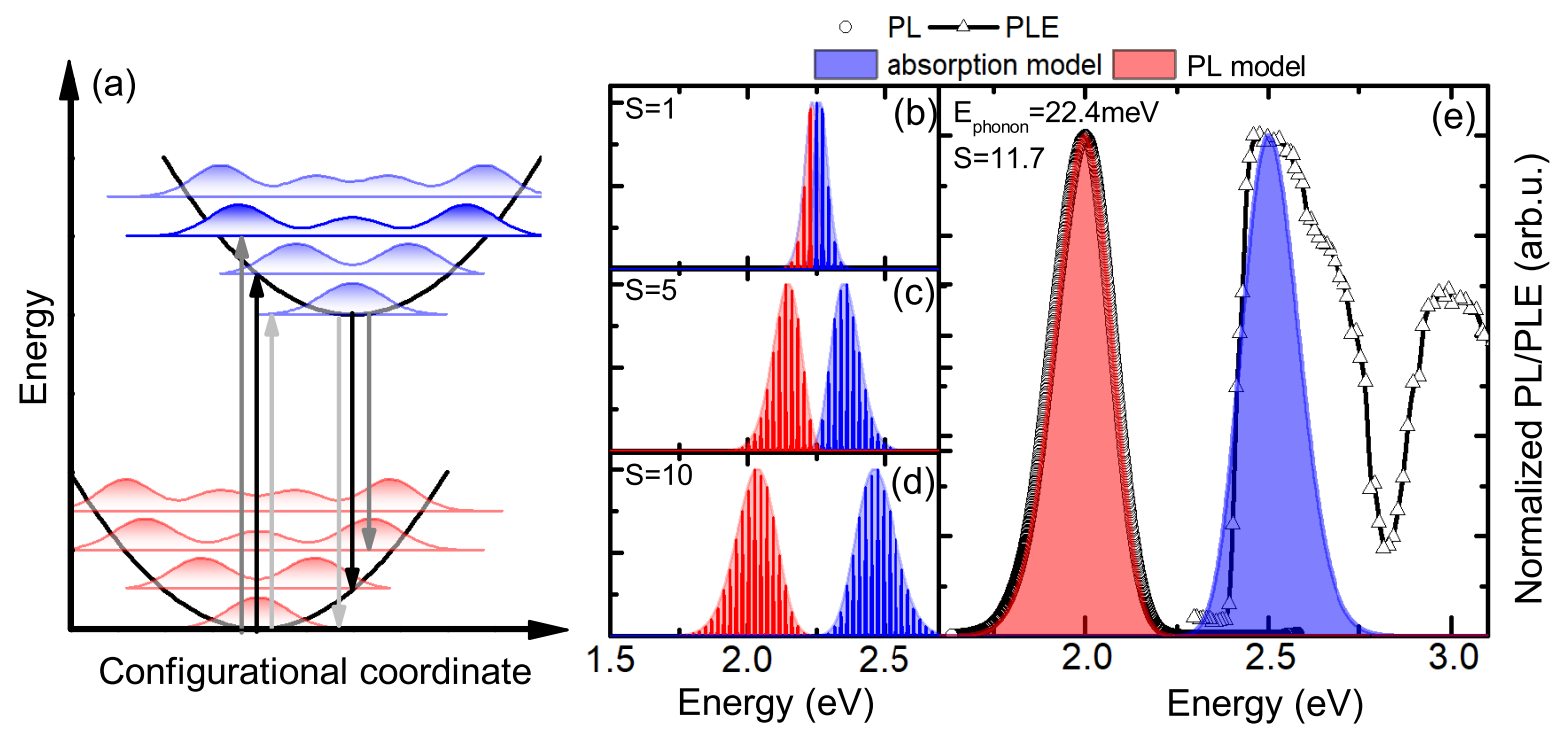

Figure 3: (a) Schematic showing the Franck-Condon model (configurational coordinate diagram). Red and blue parabole represents the harmonic potential felt by the color center in the ground and excited states. The shaded area represents spatial probability distribution of the harmonic oscillator eigen states. The arrows show transitions with 0 (light gray) 1 (black) and 2 (dark gray) phonons involved. (b) - (d) evolution of the absorption (blue) and emission spectra (red) as a function of the Huang-Rhys factor. The solid lines are spectra modelled with $1 \mathrm{meV}$ FWHM of each phonon assisted transition while the lighter colored shaded areas are calculated with $10 \mathrm{meV}$ FWHM. (e) Results of the Franck-Condon model fitting (shaded area) to the PL and PLE spectra. We assume $10 \mathrm{meV}$ broadening of each phonon transition and $22.4 \mathrm{meV}$ phonon energy.

the broadening of the PL lending strong support to the coupled color center model. Obviously the PLE spectrum is not reproduced with such a good agreement as PL since it is affected by the transfer of free carriers (excited in bandto-band transitions) to color centers, nevertheless the dominant feature is well reproduced.

The exact nature of the color centre remains an open question. It can be either intrinsic, related to the self trapping of carriers or excitons, alternatively it may be trap or defect related, $27 / 4344$ any of which can strongly deform the lattice after carrier trapping. The defects themselves can be intrinsic through the presence of defects such as $\mathrm{Ag}$ vacancies or $\mathrm{AgBi}$ anti-site defects which are calculated to act as shallow acceptors ${ }^{45}$ causing $\mathrm{Cs}_{2} \mathrm{AgBiBr}_{6}$ single crystals to become p-type, as has been concluded from photoemission measurements. $\underline{40}$ Very recently it was shown that in the case of $\mathrm{Cs}_{2} \mathrm{AgInCl}_{6}$ a strong PL emission is related to intrinsic hole self trapping at the $\mathrm{Ag}$ atom, however in the case of $\mathrm{Cs}_{2} \mathrm{AgBiBr}_{6}$ it has been sug- gested that the behaviour is defect related. $\underline{40 \mid 41}$ Our measurements suggest that defects may be a more likely explanation. The significant difference between the absorption and the PLE spectra, in particular the very sharp onset at $2.4 \mathrm{eV}$, suggests that direct excitation to the emissive state is occurring consistently with a Frank-Condon model with strong carrier trapping. By contrast the significantly different absorption spectrum is thought to be dominated by the indirect band gap leading to competition between radiative and non-radiative processes. Further evidence of this can be seen from the sharp dip in the PLE spectrum at $2.8 \mathrm{eV}$ which arises due to competition with the direct gap exciton which does not give rise to any color centre emission but corresponds to the dispersive feature in the reflectivity shown in Fig. 1, 37 The defect related origin is also in agreement with recent studies of carrier dynamics $\frac{36[40 \mid 41}{14}$ which suggest that PL is mediated through defect states.

It is worth noting that the $\mathrm{Cs}_{2} \mathrm{AgInCl}_{6} \sqrt{26}$ is 
significantly more polar than $\mathrm{Cs}_{2} \mathrm{AgInBr}_{6}$ due to the larger electronegativity differences between the $\mathrm{Ag} / \mathrm{In}$ and $\mathrm{Cl}$ atoms $(\mathrm{Cl}-\mathrm{Ag}=1.23$, $\mathrm{Cl}-\mathrm{In}=1.38$ ) compared to $\mathrm{Ag} / \mathrm{Bi}$ and $\mathrm{Br}$ $(\mathrm{Br}-\mathrm{Ag}=1.03, \mathrm{Br}-\mathrm{Bi}=0.94)$ which will enhance its probability of self-trapping. This is also consistent with the significantly larger Huang-Rhys factor of 37 observed for the Chloride compared with our value of 11.7 for the Bromide. ${ }^{26}$ A similar difference in behaviour has been reported for other metal halides such as $\mathrm{AgBr}_{1-x} \mathrm{Cl}_{x}$, where a swap over from self trapping to free excitonic behaviour has been observed as the $\mathrm{Cl}$ is replaced by $\mathrm{Br} .2728$

In conclusion, we have shown that the optical properties of $\mathrm{Cs}_{2} \mathrm{AgBiBr}_{6}$ are dominated by the strong coupling between the photoexcited carriers and phonons. The difference in the PLE and absorption spectrum demonstrates that the $\mathrm{PL}$ emission in this material originates from color centers rather than band-to-band recombination. The significant broadening of the PL together with the Stokes shift between PL and PLE peaks can be explained by a FranckCondon model indicative of a strong local lattice deformation upon excitation of the color center states which is characterized by a relatively large Huang-Rhys factor of about 12 . The significant variation of the PLE signal results from competitive pathways for carrier recombination, radiative recombination related to direct color center and, non-radiative recombination of the carriers which relax to the bottom of indirect band gap, combined also with direct gap excitonic absorption.

\section{Methods}

Experiment Samples were mounted in a helium cryostat. Optical access to the samples was provided through a quartz window. The PL and PLE measurements were performed in a back scattering configuration. PL and PLE was excited provided by femtosecond pulse (150 fs) mode-locked Ti:sapphire laser or frequency doubled output of an optical parametric oscillator, synchronously pumped by the Ti:sapphire laser. The excitation beam was focused on the sample by a $20 \mathrm{~cm}$ focal length lens. The emitted PL was collected through the same lens and redirected to a spectrometer equipped with a liquid nitrogen cooled charge-coupled device camera. The reflectivity spectrum was measured exciting with a tungsten halogen lamp. Photoacoustic spectra were measured in the microphone sensing configuration. A tunable light source consisting of a $250 \mathrm{~W}$ tungsten halogen lamp and a $0.32 \mathrm{~m}$ focal length monochromator was used to illuminate the sample mounted inside a non-resonant photoacoustic cell. The incident beam was modulated with a mechanical chopper at a frequency of $40 \mathrm{~Hz}$, passed through a quartz transmission window, and focused to a spot of $2 \mathrm{~mm} \times 1 \mathrm{~mm}$ in size. Acoustic waves arising inside the air-tight cell were detected with an electret condenser microphone producing a voltage signal further demodulated with a lock-in amplifier (Stanford Research Systems SR830).

Theory All calculations were performed using DFT as implemented in the Crystal17 code. ${ }^{46 / 47}$ The system was fully relaxed (atomic positions and lattice vectors) using PBE functional ${ }^{48}$ with van der Waals correction as proposed by Grimme $e^{49}$ and the effective core potential basis sets to account for relativistic effects. Optimization was performed using $36 k$ point mesh in the irreducible Brillouin zone following the Monkhorst-Pack approach. Raman spectra were calculated from the dielectric tensor with the Coupled Perturbed Hartree-Fock (CPHF) method, as implemented in Crystal17 code. Two models were used to represent the $\mathrm{Cs}_{2} \mathrm{AgBiBr}_{6}$ crystal structure, the cubic form

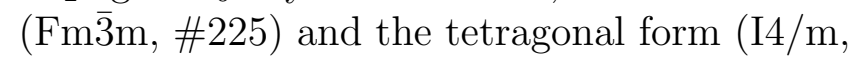
\#87) in order to understand the changes in Raman shifts with respect to the lattice vectors.

Acknowledgement This work was partially supported by the Région Midi-Pyrénées under contract MESR 13053031, BLAPHENE and STRABOT projects, which received funding from the IDEX Toulouse, Emergence program, "Programme des Investissements d'Avenir" under the program ANR11-IDEX-0002-02, reference ANR-10-LABX0037-NEXT. M.B. appreciates support from 
the Polish Ministry of Science and Higher Education within the Mobilnosc Plus program (grant no. 1648/MOB/V/2017/0). A.K. acknowledges ZIH Dresden for providing computational resources. S.J.Z. also acknowledges the support within the Etiuda 5 scholarship from National Science Centre Poland (no. 2017/24/T/ST3/00257). We acknowledge financial support from the UK Engineering and Physical Sciences Research Council (EPSRC), Grant No. EP/P033229/1, and from University college (Berman scholarship) and Balliol college (J.T. Hamilton scholarship) at Oxford University.

\section{References}

(1) Yang, W. S.; Park, B.-W.; Jung, E. H.; Jeon, N. J.; Kim, Y. C.; Lee, D. U.; Shin, S. S.; Seo, J.; Kim, E. K.; Noh, J. H. et al. Iodide management in formamidinium-lead-halide-based perovskite layers for efficient solar cells. Science 2017, 356, 1376-1379.

(2) Leijtens, T.; Bush, K. A.; Prasanna, R.; McGehee, M. D. Opportunities and challenges for tandem solar cells using metal halide perovskite semiconductors. Nature Energy 2018, 1.

(3) Sahli, F.; Werner, J.; Kamino, B. A.; Bräuninger, M.; Monnard, R.; PavietSalomon, B.; Barraud, L.; Ding, L.; Leon, J. J. D.; Sacchetto, D. et al. Fully textured monolithic perovskite/silicon tandem solar cells with $25.2 \%$ power conversion efficiency. Nature materials 2018, 1 .

(4) Stranks, S. D.; Eperon, G. E.; Grancini, G.; Menelaou, C.; Alcocer, M. J.; Leijtens, T.; Herz, L. M.; Petrozza, A.; Snaith, H. J. Electron-hole diffusion lengths exceeding 1 micrometer in an organometal trihalide perovskite absorber. Science 2013, 342, 341-344.

(5) de Quillettes, D. W.; Vorpahl, S. M.; Stranks, S. D.; Nagaoka, H.;
Eperon, G. E.; Ziffer, M. E.; Snaith, H. J.; Ginger, D. S. Impact of microstructure on local carrier lifetime in perovskite solar cells. Science 2015, 348, 683-686.

(6) Hutter, E. M.; Gélvez-Rueda, M. C.; Osherov, A.; Bulović, V.; Grozema, F. C.; Stranks, S. D.; Savenije, T. J. Directindirect character of the bandgap in methylammonium lead iodide perovskite. Nature Materials 2017, 16, 115.

(7) Huang, J.; Yuan, Y.; Shao, Y.; Yan, Y. Understanding the physical properties of hybrid perovskites for photovoltaic applications. Nature Reviews Materials 2017, 2, 17042 .

(8) Miyata, A.; Mitioglu, A.; Plochocka, P.; Portugall, O.; Wang, J. T.-W.; Stranks, S. D.; Snaith, H. J.; Nicholas, R. J. Direct measurement of the exciton binding energy and effective masses for charge carriers in organicinorganic tri-halide perovskites. Nature Physics 2015, 11, 582-587.

(9) Yang, S.; Fu, W.; Zhang, Z.; Chen, H.; Li, C.-Z. Recent advances in perovskite solar cells: efficiency, stability and lead-free perovskite. Journal of Materials Chemistry A 2017, 5, 11462-11482.

(10) Stranks, S. D.; Snaith, H. J. Metal-halide perovskites for photovoltaic and lightemitting devices. Nature Nanotechnology 2015, 10, 391.

(11) Park, N.-G. Perovskite solar cells: an emerging photovoltaic technology. Materials today 2015, 18, 65-72.

(12) Asghar, M.; Zhang, J.; Wang, H.; Lund, P. Device stability of perovskite solar cells-a review. Renewable and Sustainable Energy Reviews 2017, 77, 131-146.

(13) Christians, J. A.; Schulz, P.; Tinkham, J. S.; Schloemer, T. H.; Harvey, S. P.; de Villers, B. J. T.; Sellinger, A.; Berry, J. J.; Luther, J. M. Tailored interfaces of unencapsulated perovskite solar 
cells for: 1,000 hour operational stability. Nature Energy 2018, 3, 68.

(14) Flora, G.; Gupta, D.; Tiwari, A. Toxicity of lead: a review with recent updates. Interdisciplinary toxicology 2012, 5, 47-58.

(15) Giustino, F.; Snaith, H. J. Toward leadfree perovskite solar cells. ACS Energy Letters 2016, 1, 1233-1240.

(16) Greul, E.; Petrus, M. L.; Binek, A.; Docampo, P.; Bein, T. Highly stable, phase pure Cs $2 \mathrm{AgBiBr} 6$ double perovskite thin films for optoelectronic applications. Journal of Materials Chemistry A 2017, 5, 19972-19981.

(17) Volonakis, G.; Filip, M. R.; Haghighirad, A. A.; Sakai, N.; Wenger, B.; Snaith, H. J.; Giustino, F. Lead-free halide double perovskites via heterovalent substitution of noble metals. The journal of physical chemistry letters 2016, 7, 12541259 .

(18) Volonakis, G.; Haghighirad, A. A.; Milot, R. L.; Sio, W. H.; Filip, M. R.; Wenger, B.; Johnston, M. B.; Herz, L. M.; Snaith, H. J.; Giustino, F. Cs2InAgCl6: A new lead-free halide double perovskite with direct band gap. The journal of physical chemistry letters 2017, 8, 772-778.

(19) Bekenstein, Y.; Dahl, J. C.; Huang, J.; Osowiecki, W. T.; Swabeck, J. K.; Chan, E. M.; Yang, P.; Alivisatos, A. P. The Making and Breaking of Lead-Free Double Perovskite Nanocrystals of Cesium Silver-Bismuth Halide Compositions. Nano letters 2018, 18, 3502-3508.

(20) McClure, E. T.; Ball, M. R.; Windl, W.; Woodward, P. M. Cs2AgBiX6 (X= Br, $\mathrm{Cl}$ ): new visible light absorbing, lead-free halide perovskite semiconductors. Chemistry of Materials 2016, 28, 1348-1354.

(21) Filip, M. R.; Hillman, S.; Haghighirad, A. A.; Snaith, H. J.; Giustino, F. Band gaps of the lead-free halide double perovskites $\mathrm{Cs} 2 \mathrm{BiAgCl} 6$ and $\mathrm{Cs} 2 \mathrm{BiAgBr} 6$ from theory and experiment. The journal of physical chemistry letters 2016, 7, 2579-2585.

(22) Du, K.-z.; Meng, W.; Wang, X.; Yan, Y.; Mitzi, D. B. Bandgap Engineering of Lead-Free Double Perovskite Cs2AgBiBr6 through Trivalent Metal Alloying. Angewandte Chemie International Edition 2017, 56, 8158-8162.

(23) Slavney, A. H.; Hu, T.; Lindenberg, A. M.; Karunadasa, H. I. A bismuth-halide double perovskite with long carrier recombination lifetime for photovoltaic applications. Journal of the American Chemical Society 2016, 138, 2138-2141.

(24) Pan, W.; Wu, H.; Luo, J.; Deng, Z.; Ge, C.; Chen, C.; Jiang, X.; Yin, W.-J.; Niu, G.; Zhu, L. et al. Cs 2 AgBiBr 6 single-crystal X-ray detectors with a low detection limit. Nature Photonics 2017, 11,726 .

(25) Steele, J. A.; Pan, W.; Martin, C.; Keshavarz, M.; Debroye, E.; Yuan, H.; Banerjee, S.; Fron, E.; Jonckheere, D.; Kim, C. W. et al. Photophysical Pathways in Highly Sensitive Cs2AgBiBr6 DoublePerovskite Single-Crystal X-Ray Detectors. Advanced Materials 2018, 1804450.

(26) Luo, J.; Wang, X.; Li, S.; Liu, J.; Guo, Y.; Niu, G.; Yao, L.; Fu, Y.; Gao, L.; Dong, Q. et al. Efficient and stable emission of warm-white light from lead-free halide double perovskites. Nature 2018,

(27) Pelant, I.; Valenta, J. Luminescence spectroscopy of semiconductors; Oxford University Press, 2012.

(28) Kanzaki, H.; Sakuragi, S.; Sakamoto, K. Excitons in AgBr1-æClæ-transition of relaxed state between free and selftraffed exciton. Solid State Communications 1971, 9, 999-1002.

(29) Williams, R.; Song, K. The self-trapped exciton. Journal of Physics and Chemistry of Solids 1990, 51, 679-716. 
(30) Miyata, K.; Meggiolaro, D.; Trinh, M. T.; Joshi, P. P.; Mosconi, E.; Jones, S. C.; De Angelis, F.; Zhu, X.-Y. Large polarons in lead halide perovskites. Science advances 2017, 3, e1701217.

(31) Steele, J. A.; Puech, P.; Keshavarz, M.; Yang, R.; Banerjee, S.; Debroye, E.; Kim, C. W.; Yuan, H.; Heo, N. H.; Vanacken, J. et al. Giant Electron-Phonon Coupling and Deep Conduction Band Resonance in Metal Halide Double Perovskite. ACS nano 2018,

(32) Cortecchia, D.; Yin, J.; Bruno, A.; Lo, S.Z. A.; Gurzadyan, G. G.; Mhaisalkar, S.; Brédas, J.-L.; Soci, C. Polaron selflocalization in white-light emitting hybrid perovskites. Journal of Materials Chemistry C 2017, 5, 2771-2780.

(33) Yangui, A.; Garrot, D.; Lauret, J.-S.; Lusson, A.; Bouchez, G.; Deleporte, E.; Pillet, S.; Bendeif, E.-E.; Castro, M.; Triki, S. et al. Optical investigation of broadband white-light emission in selfassembled organic-inorganic perovskite (C6H11NH3) 2PbBr4. The Journal of Physical Chemistry C 2015, 119, 2363823647.

(34) McCall, K. M.; Stoumpos, C. C.; Kostina, S. S.; Kanatzidis, M. G.; Wessels, B. W. Strong electron-phonon coupling and self-trapped excitons in the defect halide perovskites A3M2I9 ( $\mathrm{a}=\mathrm{Cs}$, $\mathrm{Rb} ; \mathrm{M}=\mathrm{Bi}, \mathrm{Sb})$. Chemistry of Materials 2017, 29, 4129-4145.

(35) Straus, D. B.; Hurtado Parra, S.; Iotov, N.; Gebhardt, J.; Rappe, A. M.; Subotnik, J. E.; Kikkawa, J. M.; Kagan, C. R. Direct observation of electronphonon coupling and slow vibrational relaxation in organic-inorganic hybrid perovskites. Journal of the American Chemical Society 2016, 138, 13798-13801.

(36) Kentsch, R.; Scholz, M.; Horn, J.; Schlettwein, D.; Oum, K.; Lenzer, T. Exciton Dynamics and Electron-Phonon Coupling
Affect the Photovoltaic Performance of the Cs2AgBiBr6 Double Perovskite. The Journal of Physical Chemistry C 2018,

(37) Schade, L.; Wright, A. D.; Johnson, R. D.; Dollmann, M.; Wenger, B.; Nayak, P. K.; Prabhakaran, D.; Herz, L. M.; Nicholas, R.; Snaith, H. J. et al. Structural and Optical Properties of Cs2AgBiBr6 Double Perovskite. ACS Energy Letters 2019, 4, 299-305.

(38) Yang, J.; Zhang, P.; Wei, S.-H. Band Structure Engineering of Cs2AgBiBr6 Perovskite through Order-Disordered Transition: A First-Principle Study. The journal of physical chemistry letters 2017, 9, 31-35.

(39) Bartesaghi, D.; Slavney, A. H.; Gelvez-Rueda, M. C.; Connor, B. A.; Grozema, F. C.; Karunadasa, H. I.; Savenije, T. J. Charge Carrier Dynamics in Cs2AgBiBr6 Double Perovskite. The Journal of Physical Chemistry C 2018, 122, 4809-4816.

(40) Hoye, R. L.; Eyre, L.; Wei, F.; Brivio, F.; Sadhanala, A.; Sun, S.; Li, W.; Zhang, K. H.; MacManus-Driscoll, J. L.; Bristowe, P. D. et al. Fundamental Carrier Lifetime Exceeding $1 \mu \mathrm{s}$ in $\mathrm{Cs} 2 \mathrm{AgBiBr} 6$ Double Perovskite. Advanced Materials Interfaces 2018, 1800464.

(41) Steele, J. A.; Pan, W.; Martin, C.; Keshavarz, M.; Debroye, E.; Yuan, H.; Banerjee, S.; Fron, E.; Jonckheere, D.; Kim, C. W. et al. Photophysical Pathways in Highly Sensitive Cs2AgBiBr6 DoublePerovskite Single-Crystal X-Ray Detectors. Advanced Materials 0, 1804450.

(42) de Jong, M.; Seijo, L.; Meijerink, A.; Rabouw, F. T. Resolving the ambiguity in the relation between Stokes shift and Huang-Rhys parameter. Physical Chemistry Chemical Physics 2015, 17, 16959 16969. 
(43) Smith, M. D.; Karunadasa, H. I. WhiteLight Emission from Layered Halide Perovskites. Accounts of chemical research 2018, 51, 619-627.

(44) Booker, E. P.; Thomas, T. H.; Quarti, C.; Stanton, M. R.; Dashwood, C. D.; Gillett, A. J.; Richter, J. M.; Pearson, A. J.; Davis, N. J.; Sirringhaus, H. et al. Formation of long-lived color centers for broadband visible light emission in low-dimensional layered perovskites. Journal of the American Chemical Society 2017, 139, 18632-18639.

(45) Xiao, Z.; Meng, W.; Wang, J.; Yan, Y. Thermodynamic Stability and Defect Chemistry of Bismuth-Based Lead-Free Double Perovskites. ChemSusChem 2016, 9, 2628-2633.

(46) Dovesi, R.; Erba, A.; Orlando, R.; Zicovich-Wilson, C. M.; Civalleri, B.; Maschio, L.; Rerat, M.; Casassa, S.; Baima, J.; Salustro, S. et al. Quantummechanical condensed matter simulations with CRYSTAL. Wiley Interdisciplinary Reviews: Computational Molecular Science 8, e1360.

(47) Dovesi, R.; Saunders, V. R.; Roetti, C.; Orlando, R.; Zicovich-Wilson, C. M.; Pascale, F.; Civalleri, B.; Doll, K.; Harrison, N. M.; Bush, I. J. et al. CRYSTAL17 User's Manual (University of Torino, Torino, 2017). CRYSTAL17 User's Manual (University of Torino, Torino, 2017).

(48) Perdew, J. P.; Burke, K.; Ernzerhof, M. Phys. Rev. Lett. 1996, 77, 3865-3868.

(49) Grimme, S. Semiempirical GGA-type density functional constructed with a longrange dispersion correction. J. Comp. Chem. 2006, 27, 1787-1799. 